\title{
Extraction and Separation of Phycocyanin from Spirulina using Aqueous Two-Phase Systems of Ionic Liquid and Salt
}

\author{
Xifeng Zhang', Fenqin Zhang ${ }^{1}$, Guanghong Luo ${ }^{2, *}$, Shenghui Yang ${ }^{2}$, Danxia Wang ${ }^{2}$ \\ ${ }^{1}$ The College of Agriculture and Biotechnology (CAB), Hexi University, Zhangye, P.R. China \\ ${ }^{2}$ Kaiyuan Bio-tech Development Center, Hexi University, Zhangye, P.R. China \\ *Corresponding author: 464690924@qq.com
}

Received December 02, 2014; Revised December 11, 2014; Accepted December 30, 2014

\begin{abstract}
To explore a new and simple rapid extraction and purification technique for phycocyanin, an ionic liquids(ILs)-based aqueous two-phase system(ATPS) was developed for the purification of phycocyanin from Spirulina extracts. Effects of various process parameters such as the concentrations of [Bmim] $\mathrm{Cl}$, the concentrations of $\mathrm{KH}_{2} \mathrm{PO}_{4}$, the concentrations of crude phycocyanin, the system $\mathrm{pH}$ and the temperature on partitioning of phycocyanin were evaluated. The obtained data indicated that phycocyanin was preferentially partitioned into the ILs-rich phase and the ATPS composed of $23 \%(\mathrm{w} / \mathrm{w})$ [Bmim]Cland $29 \%(\mathrm{w} / \mathrm{w}) \mathrm{KH}_{2} \mathrm{PO}_{4}$ at $30^{\circ} \mathrm{C}$ and $\mathrm{pH} 7.0$ showed good selectivity on phycocyanin. Under the optimum conditions, phycocyanin with a purity of 3.98 and yield of about $90.23 \%$ was obtained. Therefore, ILs-based ATPS was an effective method for partitioning and recovery of phycocyanin from Spirulina extracts.
\end{abstract}

Keywords: Ionic liquid, Aqueous two-phase system, phycocyanin, Purification

Cite This Article: Xifeng Zhang, Fenqin Zhang, Guanghong Luo, Shenghui Yang, and Danxia Wang, "Extraction and Separation of Phycocyanin from Spirulina using Aqueous Two-Phase Systems of Ionic Liquid and Salt.” Journal of Food and Nutrition Research, vol. 3, no. 1 (2015): 15-19. doi: 10.12691/jfnr-3-1-3.

\section{Introduction}

Cultivation of Spirulina microalga is an effective process for obtaining several valuable biochemicals, such as polysaccharides [1], $\gamma$-linolenicacid [2], $\beta$-carotene [3], chlorophylla [4] and phycobiliproteins [5]. Phycobiliproteins, which are brightly colored pigments, function as a receiver of light for driving photosynthesis in the Spirulina microalga [6]. Microalgal phycobiliproteins are classified into three major groups: phycoerythrin, allophycocyanin, and phycocyanin [6]. The predominant pigment in the phycobiliprotein family is phycocyanin [7]. Phycocyanin is commonly used as a natural colorant in food and cosmetic industries because it is inherently blue [6]. Moreover, it can be incorporated into health foods because of its physiological properties, such as antioxidant, antiinflammatory, and hepatoprotective activities [8,9]. Because of these benefits, numerous researchers have focused on developing efficient processes for mass production of phycocyanin-producing strains $[10,11]$ and extraction of phycocyanin from microalgae [5,12].

There are some difficulties in phycocyanin extraction because of multilayered cell walls and large amounts of contaminants. Several methods have been reported for successful separation of phycocyanin [13-17] but these methods comprised multiple steps and are time consuming, which may lead to increase in production costs and limit their widespread application.

Alternatively, Aqueous two-phase systems (ATPS) is regarded as a simple and environmentally friendly separation system. ATPS also offers many advantages, such as a low process time, low energy consumption, and an environment biocompatible to the biomolecule because each phase contains 70-90\% water, which means that biomolecules will not be denatured. Hence, ATPS has been recognized as an efficient and economical method for the separation of biomolecules [18].

In recent years, as a green solvent, ionic liquid (IL) has been widely applied in various fields of chemistry. ILs are defined as salts with a melting point below $100^{\circ} \mathrm{C}$ and they are composed of organic cations and various anions. Compared with water and organic solvents, they exhibit a negligible volatility, non-flammability, a wide electro chemical window, high thermal and chemical stability, and strong solubility power [19,20]. The IL-ATPS has advantages with combination of IL and ATPS, such as negligible viscosity, little emulsion formation, without volatile organic solvent, quick phase separation, high extraction efficiency, and gentle biocompatible environment [21]. Therefore, this technique provided a biocompatible environment for the moderate extraction and purification of biological substances, such as proteins, enzymes and nucleic acids. Moreover, IL-ATPS allows the separation, purification and the concentration to be 
integrated into one step. Thus, bioseparation engineering has desirable developing prospects in the IL-ATPS.

In the present study, IL-ATPSs formed by adding inorganic salt $\mathrm{K}_{2} \mathrm{HPO}_{4}$ into aqueous [Bmim]Cl were employed to separate phycocyanin from Spirulina. The extraction efficiency of phycocyanin and the influence of different process parameters (e.g., inorganic salt concentration, IL mass, crude phycocyanin concentration, $\mathrm{pH}$, and temperature) were analyzed in detail. The aim of this study was to develop an extraction and purification method for phycocyanin from Spirulina by IL-ATPS.

\section{Materials and Methods}

\subsection{Materials and Reagents}

Spirulina was acquired from Kaiyuan Bio-tech Development Center (Zhangye, China). The salts and other analytical grade chemicals were purchased from the National Institute for the Control of Pharmaceutical and Biological Products (Beijing, China) Water used for preparation of aqueous solutions was from a Millipore Direct-Q Water system(resistivity, $18.2 \mathrm{M} \Omega \cdot \mathrm{cm}$ ).

\subsection{Preparation of Crude Phycocyanin}

Spirulina platensis dried powder were suspended at $0.06 \mathrm{~g} / \mathrm{mL}$ in $20 \mathrm{mmol} / \mathrm{L}$ Tris-HCl buffer (contain 10 mmol/L EDTA, pH6.5). Phycocyanin was extracted by repeated freezing $\left(-20^{\circ} \mathrm{C}\right)$ and thawing at room temperature until the blue color becomes in acetate buffer. Cell debris was removed by centrifugation at $5000 \mathrm{rpm}$ for 10 min and the extract thus obtained was termed as crude extract.

\subsection{Preparation of ILATPS}

ATPSs were prepared in $10 \mathrm{~mL}$ centrifuge tubes by adding the appropriate amount of $[\mathrm{Bmim}] \mathrm{Cl}, \mathrm{K}_{2} \mathrm{HPO}_{4}$ and crude extract. Distilled water was used to bring the final weight of the system to $10 \mathrm{~g}$. Another mixture with the same phase components but without crude phycocyanin was prepared as a blank to avoid interference. The mixture was stirred well to make the $\mathrm{K}_{2} \mathrm{HPO}_{4}$ dissolve completely. The phase-separation was speeded up by centrifugation at a rolling speed of $4000 \mathrm{rpm}$ for $5 \mathrm{~min}$, two clear phases formed. The partitioning experiments were done at room temperature $\left(25^{\circ} \mathrm{C}\right)$, the top phase was mainly composed of IL and phycocyanin, and the bottom phase was the saltrich solution containing impurities. The volume of each phase was noted down.

\subsection{Effect of Concentration of [Bmim]Cl on Partitioning of Phycocyanin}

To study the effect of concentration of $[\mathrm{Bmim}] \mathrm{Cl}$, [Bmim]Cl of different mass were mixed with $29 \%(\mathrm{w} / \mathrm{w}) \mathrm{KH}_{2} \mathrm{PO}_{4}$ to form ATPS Partitioning was performed as described previously.

The phycocyanin concentration during the extraction process was determined according to the procedure used by Chen et al. [22]. The equilibrium concentrations of phycocyanin in the top and bottom phases were determined spectrophotometrically at 620 and $652 \mathrm{~nm}$ using an SP-721 spectrophotometer (Shanghai Spectrum Instrument Co.LTD, China)., respectively. The phycocyanin concentration was estimated using Eq. (1)

$$
C(\mathrm{mg} / \mathrm{mL})=\frac{O D 620-0.474 O D 650}{5.34}
$$

where $\mathrm{C}$ is the phycocyanin concentration $(\mathrm{mg} / \mathrm{mL})$, and OD620 and OD652 are the optical density of the sample at 620 and $652 \mathrm{~nm}$, respectively.

The phase ratio $(\mathrm{R})$ is defined as

$$
R=\frac{V_{i}}{V_{\mathrm{s}}}
$$

where $V_{i}$ and $V_{s}$ stand for the volume of the IL-rich phase and salt-rich phase, respectively.

The partition coefficient $(\mathrm{K})$ is defined as

$$
K=\frac{C i}{C s}
$$

where $C_{i}$ and $C_{s}$ are the concentrations of phycocyanin in the IL-rich phase and salt-rich phase, respectively.

The extraction efficiency (E) of phycocyanin in the ILrich phase is determined from the

$$
E=\frac{R K}{1+R K}
$$

The purity of phycocyanin was defined as the ratio of absorbance at $620 \mathrm{~nm}$ to $280 \mathrm{~nm}$, wherein $\mathrm{OD}_{620}$ is the maximum absorbance of phycocyanin and $\mathrm{OD}_{280}$ is the absorbance of total proteins [23]

\subsection{Effect of Concentration of $\mathrm{K}_{2} \mathrm{HPO}_{4}$ on Partitioning of Phycocyanin}

To study the effect of $\mathrm{K}_{2} \mathrm{HPO}_{4}$ on partitioning of the phycocyanin in ATPS, $\mathrm{K}_{2} \mathrm{HPO}_{4}$ at different concentrations (23, 25, 27, 29 and 31\% w/w) were mixed with 23\%(w/w) [Bmim] Cl in ATPS. Based on E and Purity, the ATPS rendering the most effective partitioning was chosen for further study.

\subsection{Effect of Crude Phycocyanin Concentration on Partitioning of Phycocyanin}

To study the effect of crude phycocyanin on partitioning of the phycocyanin in ATPS, crude phycocyanin at different concentrations (1 $\mathrm{mg} / \mathrm{mL}, 3$ $\mathrm{mg} / \mathrm{mL}, 5 \mathrm{mg} / \mathrm{mL}, 7 \mathrm{mg} / \mathrm{mL}$ and $9 \mathrm{mg} / \mathrm{mL}$ ) were mixed with $23 \%(\mathrm{w} / \mathrm{w})[\mathrm{Bmim}] \mathrm{Cl}$ and $29 \%(\mathrm{w} / \mathrm{w}) \mathrm{KH}_{2} \mathrm{PO}_{4}$ in ATPS. Based on E and Purity, the ATPS rendering the most effective partitioning was chosen for further study.

\subsection{Effect of $\mathbf{p H}$ on Partitioning of Phycocyanin}

In order to investigate the influence of $\mathrm{pH}$, an IL-based ATPS consisting of $23 \%(\mathrm{w} / \mathrm{w})[\mathrm{Bmim}] \mathrm{Cl}$ and $29 \%(\mathrm{w} / \mathrm{w}) \mathrm{KH}_{2} \mathrm{PO}_{4}$ was prepared. The $\mathrm{pH}$ of the systems was adjusted by $\mathrm{HCl}$ or $\mathrm{NaOH}$. Based on $\mathrm{E}$ and Purity, the ATPS rendering the most effective partitioning was chosen for further study. 


\subsection{Effect of Temperature on Partitioning of Phycocyanin}

The model system to evaluate the effect of temperature on phycocyanin partitioning was prepared as described above. Phase separation was induced by storing the samples in a water bath for 1-2 h at a temperature of 25 , $30,35,40$ and $45^{\circ} \mathrm{C}$, respectively. The performance of partitioning were measured to study the effect of temperature.

\subsection{Sodium Dodecyl Sulphate-Gel Electrophoresis}

Sodium dodecyl sulphate-polyacrylamide gel electrophoresis (SDS-PAGE) was carried out by following Deutscher's method [24], using a 30\% polyacrylamide slab gel. Electrophoresis was run at $50 \mathrm{~V}, 12.5 \mathrm{~mA}$, for 3$4 \mathrm{~h}$. The gel was stained with a solution that was 0.05\%(w/w) Coomassie Brilliant Blue R250, 50\%(v/v) methanol and $12 \%(\mathrm{v} / \mathrm{v})$ acetic acid. The gel was destained using a buffer that was identical to the staining solution except that it contained no Coomassie Brilliant Blue.

\section{Results and Discussion}

\subsection{Effect of Concentration of [Bmim]Cl on Partitioning of Phycocyanin}

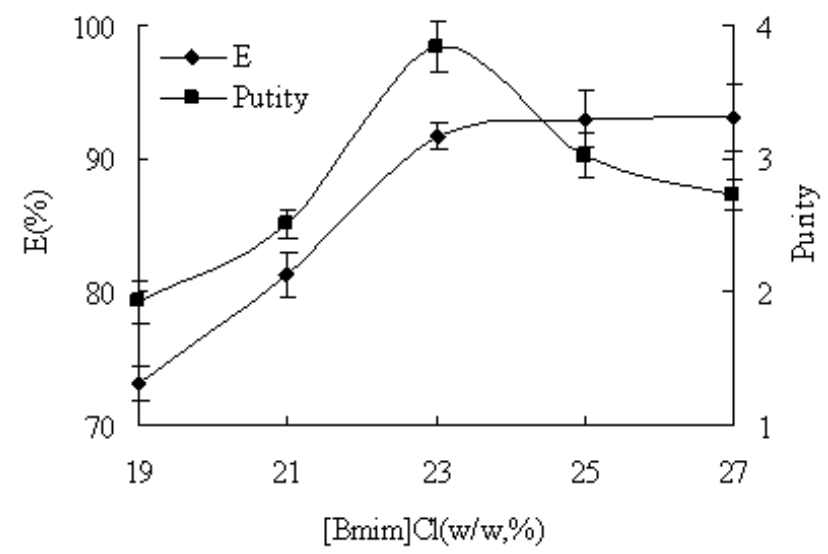

Figure 1. The influence of different concentration of [Bmim]Cl in ATPS on the extraction efficiency and purity of phycocyanin

The effect of $[\mathrm{Bmim}] \mathrm{Cl}$ amount in the system on the extraction efficiency and purity of phycocyanin is shown in Figure 1a. The extraction efficiency of phycocyanin increased with increasing [Bmim]Cl amount when the concentration of $\mathrm{KH}_{2} \mathrm{PO}_{4}$ was controlled at $29 \%$ (w/w).The purity of phycocyanin in the top phase increased as the concentration of $[\mathrm{Bmim}] \mathrm{Cl}$ increased till the concentration reached $23 \%$. At the concentration above 23\%, The extraction efficiency still increased but purity of phycocyanin decreased. This observation can be attributed to the advanced amount of [Bmim]Cl. In general, imidazolium cation of the ILs has an aromatic $\pi$ system. It appears that the $\pi-\pi$ interaction between the imidazolium cation and the aromatic residues of the proteins was a possible driving force for the extraction of proteins [25]. The probably reason was that more phycocyanin was partitioned to the top phase as the concentration increased to a higher value while residual proteins partitioned to the top phase also increased. As a result purity of phycocyanin in top phase decreased.

\subsection{Effect of $\mathrm{KH}_{2} \mathrm{PO}_{4}$ on Partitioning of Phycocyanin}

The effect of $\mathrm{KH}_{2} \mathrm{PO}_{4}$ concentration on the extraction efficiency and purity of phycocyanin is shown in Figure 2. The extraction efficiency and purity of phycocyanin showed an increased trend with increasing $\mathrm{KH}_{2} \mathrm{PO}_{4}$ concentration from $23 \%$ to $29 \%$. The maximum extraction efficiency and purity of phycocyanin were obtained when the concentration of $\mathrm{KH}_{2} \mathrm{PO} 4$ was controlled at $29 \%$. This result may be attributed to the salt-out effect. The hydrophobicity of the bottom phase increased with increasing inorganic salt concentration, in which the solubility of phycocyanin decreased because of competition between salt ions and phycocyanin for water molecules through intermolecular hydrogen bonds [26]. This competition yields less phycocyanin dissolved into the salt-rich phase, thereby lowering the extraction efficiency and purity.

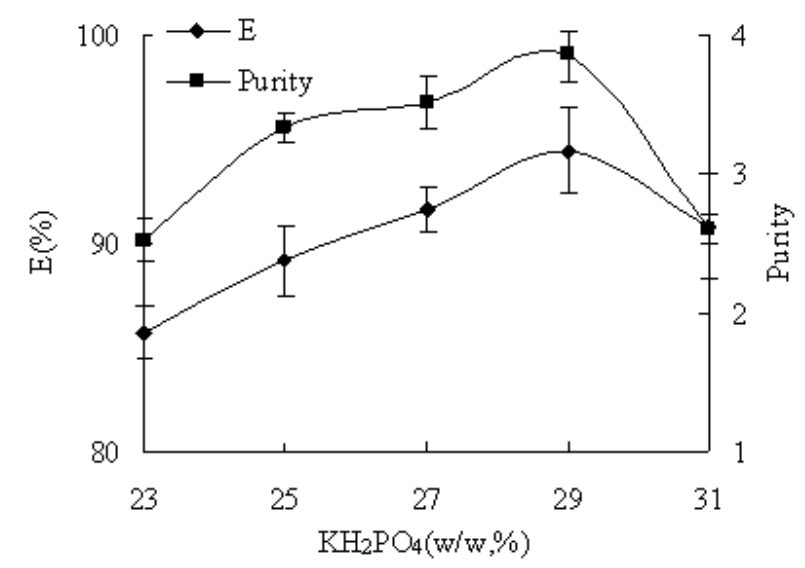

Figure 2. The influence of different concentration of $\mathrm{KH}_{2} \mathrm{PO}_{4}$ in ATPS on the extraction efficiency and purity of phycocyanin

\subsection{Effect of Crude Phycocyanin Concentration on Partitioning of Phycocyanin}

The effect of crude phycocyanin concentration on the extraction efficiency and purity is presented in Figure 3. The extraction efficiency and purity of phycocyanin showed an increased trend with increasing crude phycocyanin concentration from 1 to $3 \mathrm{mg} / \mathrm{mL}$. However, the extraction efficiency and purity of phycocyanin were decreased slightly with further increase of crude phycocyanin concentration from 3 to $5 \mathrm{mg} / \mathrm{mL}$. This phenomenon could be attributed to the fact that increasing the crude phycocyanin concentration causes the aggregation of phycocyanin molecules and the enhancement of intra-molecular hydrogen bonds, which weaken the interactions between phycocyanin and water molecules. Moreover, excessive addition of crude phycocyanin caused accumulation at the interface, which negatively affected mass transfer. The extraction system has a limited ability of extraction with a certain amount of ionic liquid. These results suggest that the crude phycocyanin concentration should be $3 \mathrm{mg} / \mathrm{mL}$. 


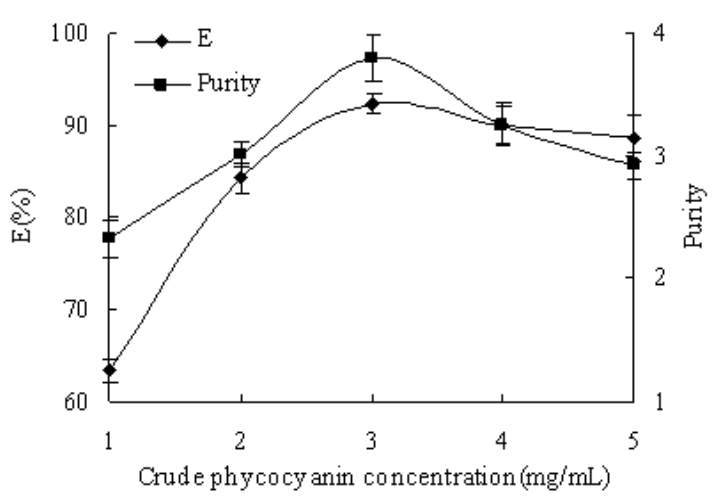

Figure 3. The influence of different concentration of crude phycocyanin in $[\mathrm{Bmim}] \mathrm{Cl}-\mathrm{KH}_{2} \mathrm{PO}_{4}$ ATPS on the extraction efficiency and purity of phycocyanin

\subsection{Effect of $\mathbf{p H}$ on Partitioning of Phycocyanin}

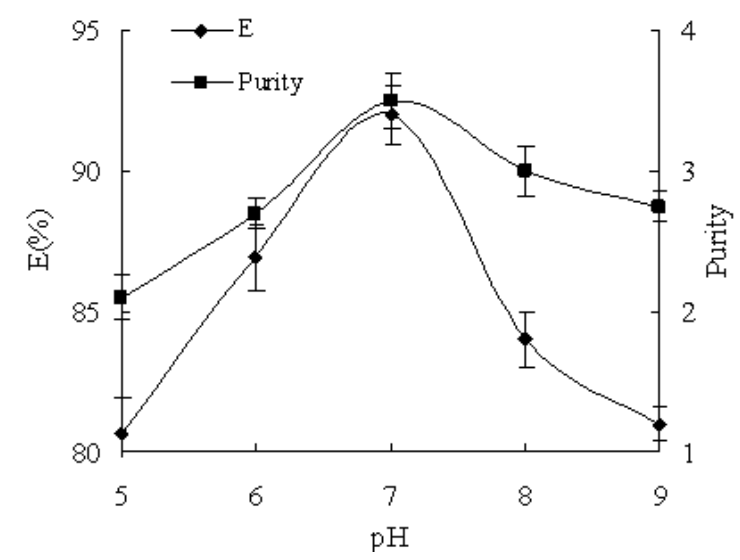

Figure 4. Effect of $\mathrm{pH}$ in $[\mathrm{Bmim}] \mathrm{Cl}-\mathrm{KH}_{2} \mathrm{PO}_{4}$ ATPS on the extraction efficiency and purity of phycocyanin

From Figure 4, the highest extraction efficiency and purity shown by ILATPS was at $\mathrm{pH} 7$. This showed that at neutral $\mathrm{pH}$ phycocyanin partition better behaviour compared to acidic or alkaline conditions. $\mathrm{pH}$ changed the partition behaviour of phycocyanin as evidenced by lower purity on systems with $\mathrm{pH} 5, \mathrm{pH} 6.0, \mathrm{pH} 8.0$ and $\mathrm{pH} 9.0$ compared to $\mathrm{pH}$ 7.0. The $\mathrm{pI}$ of phycocyanin was 4.3 . Above the isoelectric point (pI), phycocyanin with negative charge prefer the top phase in ATPS, while proteins with positive charge normally partition selectively to the bottom phase. The manipulation of $\mathrm{pH}$ in ILATPS correlated with electrochemical interactions between protein and solvent in the system. These electrochemical interactions played an important role in partition behaviour of phycocyanin [27]. Another reason was hydrophobic interaction. The driving force originated from the hydrophobic interactions between the exposed amino acid residues on the surface of the protein and the imidazole ring of the IL cations. Interaction of the two substances contained an aromatic ring with $\pi$ electrons, and $\pi-\pi$ conjugation was produced when the imidazole and amino groups were close to each other. Thus, electrostatic interactions and $\pi-\pi$ conjugation between the charged groups in proteins and the ionic group of ILs have significant functions in determining the dependence of the extraction efficiency and purity of proteins on the $\mathrm{pH}$ of aqueous solutions [28]. Therefore, the $\mathrm{pH}$ should be controlled at 7 in this study.

\subsection{Effect of the Temperature on Partitioning of Phycocyanin}

To further confrim the extraction temperature studied on the influence of extraction eficiency and purity of phycocyanin in IL-based aqueous two-phase systems, the phycocyanin content on its distribution behavior was also studied. In light of Figure 5, as the temperature increased from 25 to $30^{\circ} \mathrm{C}$, the extraction efficiency of the phycocyanin increased from $90.15 \%$ to $93.03 \%$. When the temperature was kept at $30^{\circ} \mathrm{C}$ or higher, the extraction yield and purity decreased correspondingly. The possible reason for this phenomenon is that the increased extraction temperature could reduce the viscosity of the ionic liquid, enhance the solvent solubility and diffusion capacity. However, when the temperature continues to rise, the extraction rate was reduced. On the one hand, it means that the temperature was high to destroy the hydrogen bonding interaction between the surface water of protein and amino acid residue. On the other hand, as the temperature rises, the extraction rate is reduced resulting in a tendency for the liquid to be homogeneous. So the extraction was carried out at $30^{\circ} \mathrm{C}$ because of the relatively high extraction yield and purity.

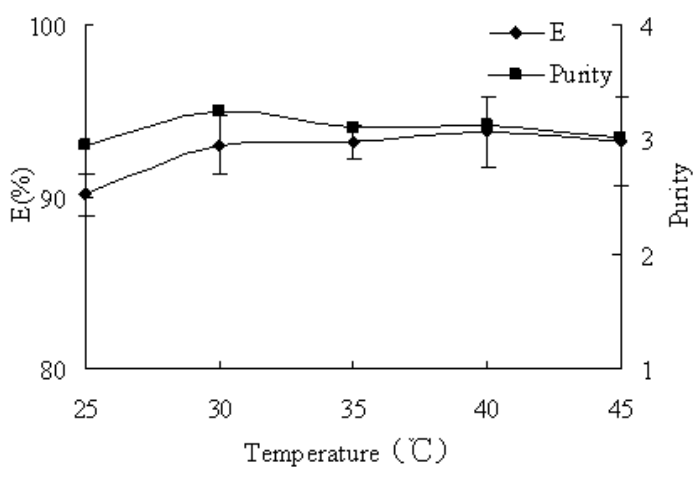

Figure 5. Effect of temperature in $[\mathrm{Bmim}] \mathrm{Cl}-\mathrm{KH}_{2} \mathrm{PO}_{4}$ ATPS on the extraction efficiency and purity of phycocyanin

\subsection{SDS-PAGE}

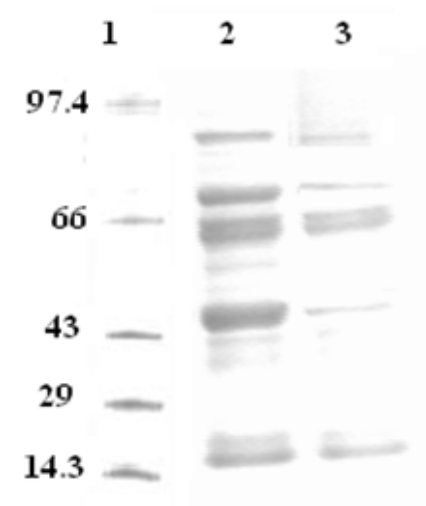

Figure 6. SDS-PAGE of phycocyanin purified by aqueous two-phase extraction: Lane 1- Marker; Lane 2- Crude extract; Lane 3- Top phase

The purity of phycocyanin obtained from ILATPS was confirmed using SDS-PAGE as shown in Figure 6. Lane 1 indicated the molecular marker, while lane 2 was the crude extract of phycocyanin and lane 3 was the phycocyanin after ILATPS extraction. From the SDSPAGE, an increase in the purity of phycocyanin is observed after the ILATPS extraction. 


\subsection{Recovery of IL}

Considering that ILs can impact on the water ecosystems and ILs remain very expensive in comparison with the conventional extractants, it is significantly important to recycle ILs in using IL-based ATPS, especially when dealing with their application on a large scale and in related wastewater streams [28].

In this study, a large number of [ $\mathrm{Bmim}] \mathrm{Cl}$ remain in the IL-rich phase after ILATPS extraction. In the light of previous study [27], the IL-rich phase containing [Bmim]Cl was initially concentrated to remove water under reduced pressure, and then extracted into $\mathrm{CH}_{2} \mathrm{Cl}_{2}$ solution. [Bmim]Cl can be recycled after removing $\mathrm{CH}_{2} \mathrm{Cl}_{2}$.

\section{Conclusions}

Purification of phycocyanin from the crude extract using $[\mathrm{Bmim}] \mathrm{Cl} / \mathrm{KH}_{2} \mathrm{PO}_{4}$ ATPS is reported for the first time. During this study, a systematic approach was used to find the optimized conditions to purify phycocyanin. The process parameters involved in the purification of phycocyanin were discussed and their optimization was described in detail. A method for purification of phycocyanin in an ATPS was proposed. With a $23 \%[\mathrm{Bmim}] \mathrm{Cl}$ and $29 \% \mathrm{KH}_{2} \mathrm{PO}_{4}$ ATPS, phycocyanin with a purity of 3.98 and yield of about $90.23 \%$ was obtained. This method enhances both the purity and the yield of the phycocyanin beyond that obtained by the conventional salting-out step. Experimental results obtained here demonstrated the feasibility of an ATPS for the purification of phycocyanin.

\section{Acknowledgement}

The authors would like to acknowledge the financial support by the Gansu Provincial Foundation for Technology Based Firms (No.1047GCCG001).

\section{References}

[1] G.G. Choi, M.S. Bae, C.Y. Ahn, H.M. Oh, Induction of axenic culture of Arthrospira (Spirulina) platensis based on antibiotic sensitivity of contaminating bacteria, Biotechnol. Lett. 30 (2008) 87-92.

[2] M.G. Sajilata, R.S. Singhal, M.Y. Kamat, Fractionation of lipids and purification of gamma-linolenic acid (GLA) from Spirulina platensis, Food Chem. 109 (2008) 580-586.

[3] T. Gireesh, A. Jayadeep, K.N. Rajasekharan, V.P. Menon, M. Vairamany, G. Tang, P.P. Nair, P.R. Sudhakaran, Production of deuterated beta-carotene by metabolic labelling of Spirulina platensis, Biotechnol. Lett. 23 (2001) 447-449.

[4] H.B. Chen, J.Y. Wu, C.F. Wang, C.C. Fu, C.J. Shieh, C.I. Chen, C.Y. Wang, Y.C. Liu, Modeling on chlorophyll a and phycocyanin production by Spirulina platensis under various light-emitting diodes, Biochem. Eng. J. 53 (2010) 52-56.

[5] T.Silveira, J.F.M.Burkert, J.A.V.Costa, C.A.V.Burkert, S.J.Kalil, Opti mization of phycocyanin extraction from Spirulina platensis using factorial design, Bioresour. Technol. 98 (2007) 1629-1634.

[6] M.C.Santiago-Santos,T.Ponce-Noyola,R.Olvera-Ramirez, J.Ortega-Lopez, R.O. Canizares-Villanueva, Extraction and purification of phycocyanin from Calothrix sp, Process Biochem. 39 (2004) 2047-2052.

[7] G. Patil, K. Raghavarao, Aqueous two phase extraction for purification of C-phycocyanin, Biochem. Eng. J. 34 (2007) 156-164.
[8] V.B. Bhat, K.M. Madyastha, C-Phycocyanin: a potent peroxyl radical scavenger in vivo and in vitro, Biochem. Biophys. Res. Commun. 275 (2000) 20-25.

[9] M.C. Reddy, J. Subliashini, S.V.K. Mahipal, V.B. Bhat, P.S. Reddy, G. Kiranmai, K.M. Madyastha, P. Reddanna, CPhycocyanin, a selective cyclooxygenase-2 inhibitor, induces apoptosis in lipopolysaccharide-stimulated RAW 264.7 macrophages, Biochem. Biophys. Res. Commun. 304 (2003) 385-392.

[10] M.G. de Morais, J.A.V. Costa, Carbon dioxide fixation by Chlorella kessleri, C-vulgaris, Scenedesmus obliquus and Spirulina sp cultivated in flasks and vertical tubular photobioreactors, Biotechnol. Lett. 29 (2007) 1349-1352.

[11] C.Y. Wang, C.C. Fu, Y.C. Liu, Effects of using light-emitting diodes on the cultivation of Spirulina platensis, Biochem. Eng. J. 37 (2007) 21-25.

[12] S.G. Yan, L.P. Zhu, H.N. Su, X.Y. Zhang, X.L. Chen, B.C. Zhou, Y.Z. Zhang, Single-step chromatography for simultaneous purification of C-phycocyanin and allophycocyanin with high purity and recovery from Spirulina (Arthrospira) platensis, J. Appl. Phycol. 23 (2011) 1-6.

[13] Boussiba, S., \& Richmond,A.E. (1979). Isolation and characterization of phycocyanins from the blue-green alga Spirulina platensis. Archives of Microbiology, 120, 155-159.

[14] Ranjitha, K., \& Kaushik, B. D. (2005). Purification of phycobiliproteins from Nostoc muscorum. Journal of Scientific and Industrial Research, 64, 372-375.

[15] Eriksen, N. T. (2008).Production of phycocyanin-a pigment with applications in biology, biotechnology, foods and medicine. Applied Microbiology and Biotechnology, 80, 1-14.

[16] Yan, S., Zhu, L., Su, H., Zhang, X., Chen, X., Zhou, B., \& Zhang, Y. (2011). Single-step chromatography for simultaneous purification of C-phycocyanin and allophycocyanin with high purity and recovery from Spirulina (Arthrospira) platensis. Journal of Applied Phycology, 23, 1-6.

[17] Tchernov, A. A., Minkova, K. M., Houbavenska, N. B., \& Kovacheva, N. G. (1999). Purification of phycobiliproteins from Nostoc sp. by aminohexyl-sepharose chromatography. Journal of Biotechnology, 69, 69-73.

[18] Aguilar, O., \& Rito-Palomares, M. (2010). Aqueous two - phase systems strategies for the recovery and characterization of biological products from plants. Journal of the Science of Food and Agriculture, 90 (9), 1385-1392.

[19] S. Dreyer, P. Salim, U. Kragl, Driving forces of protein partitioning in an ionic liquid-based aqueous two-phase system, Biochem. Eng. J. 46 (2009) 176-185.

[20] J.A.P. Coutinho, C.M.S.S. Neves, S.P.M. Ventura, M.G. Freire, I.M. Marrucho, Evaluation of cation influence on the formation and extraction capability of ionic-liquid-based aqueous biphasic systems, J. Phys. Chem. B 113 (2009) 5194-5199.

[21] Y.C. Pei, J.J. Wang, K. Wu, X.P. Xuan, X.J. Lu, Ionic liquidbased aqueous two-phase extraction of selected proteins, Sep. Purif. Technol. 64 (2009) 288-295.

[22] H.B. Chen, J.Y. Wu, C.F. Wang, C.C. Fu, C.J. Shieh, C.I. Chen, C.Y. Wang, Y.C. Liu, Modeling on chlorophyll a and phycocyanin production by Spirulina platensis under various light-emitting diodes, Biochem. Eng. J. 53 (2010) 52-56.

[23] Boussiba S,Richmond A E(1979)Isolation and purification of phycocyanins from the blue green algae Spirulina platensis. Arch Microbiol 120: 155-159.

[24] Deutscher M (1990) Guide to protein purification, vol 182. Methods in enzymology. Academic Press, New York.

[25] novak U, Pohar a, Plazl l, Znidarsic-Plazl P (2012) Ionic liquidbased aqueous two-phase extraction within a microchannel system. Sep Purif Technol 97: 172-178.

[26] Ma CH, liu TT, Yang l, Zu Yg, Chen XQ, Zhang l, Zhang Y,Chao CJ (2011) Ionic liquid-based microwave-assisted extraction of essential oil and biphenyl cyclooctene lignans from Schisandra chinensis Baill fruits. J Chromatogr a 1218: 8573-8580.

[27] M.G. Freire, A.F.M. Cláudio, J.M.M. Araújo, J.A.P. Coutinbo, I.M. Marrucho, J.N.C.Lopes, L.P.N. Rebelo, Aqueous biphasic systems: a boost brought about byusing ionic liquids, Chem. Soc. Rev. 41 (2012) 4966-4995.

[28] F.Z. Deng, D.F. Guo, Extraction separation of bovine serum albumin in ionic liquid aqueous two-phase system, Chin. J. Anal. Chem. 34 (2006) 1451-1453. 